\title{
ENGINEERING ANALYSIS OF THE APT CRYOMODULES*
}

\author{
B.M. Campbell, K.C.D. Chan, M.J. Fagan", \\ R.A. Valicenti, and J.A. Waynert, LANL
}

\section{Abstract}

The superconducting radio frequency (SRF) part of the Accelerator Production of Tritium (APT) linac will accelerate a $100-\mathrm{mA}$ proton beam from $217 \mathrm{MeV}$ to 1700 $\mathrm{MeV}$. Since SRF cavities can accept protons over a wide velocity range, cavities with only two different betas are required; however, three different length cryomodules are required. A modular design was adopted that will reduce the engineering and design effort to produce these cryomodules. A final design of the APT Engineering Development and Demonstration (ED\&D) cryomodule for a two-cavity $\beta=0.64$ cryomodule has been completed, and a single cryomodule will be fabricated by industry next year. The cavities will be cooled in a $2.15-\mathrm{K}$ superfluid helium bath similar to the Continuous Electron Beam Accelerator (CEBAF) system. What sets the APT cryomodules apart is the high radio frequency (RF) power that must be delivered to the cavities. The RF losses in the cavities and power couplers place a large heat load on the central helium liquifier. Minimizing these loads required extensive iterations of the power coupler cooling schemes and thermal shield. A spoke support arrangement was developed to keep the beam centerline fixed and to minimize forces acting on the helium vessel/cavity during cool down. Laminar flow through the cryomodule during clean room assembly dictated the use of a vacuum vessel with large top and bottom openings. Analyses were performed to ensure structural integrity under vacuum loading, while also minimizing vessel deflections which could impact beam centerline positioning.

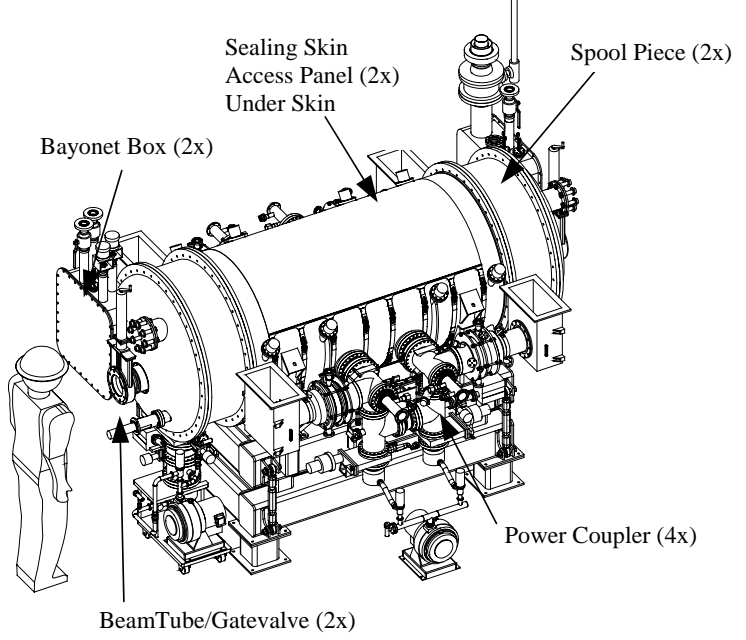

Figure 1: $\beta=0.64$ two cavity ED\&D cryomodule

\footnotetext{
* Work supported by US Department of Energy

\# E-Mail: fagan@lanl.gov
}

The structural/thermal analysis used to optimize the cryomodule design will be presented in this paper.

\section{INTRODUCTION}

A cryomodule containing two $\beta=0.64$ cavities ( $\beta=$ particle velocity/speed of light) is being designed at Los Alamos as part of the Engineering Development and Demonstration (ED\&D) program. The other types of cryomodules will be designed by General Atomics, one type for three $\beta=0.64$ cavities and another type for four $\beta=0.82$ cavities. A modular approach was adopted that will reduce the total engineering and design effort required to produce these cryomodules. Detailed descriptions for the cryomodule designs have been published [1]. The $\beta=0.64 \mathrm{ED} \& D$ cryomodule is pictured in Figures 1 and 2. The length is $3.3 \mathrm{~m}$, width is $3 \mathrm{~m}$, diameter is $1 \mathrm{~m}$, and the weight is $3000 \mathrm{~kg}$.

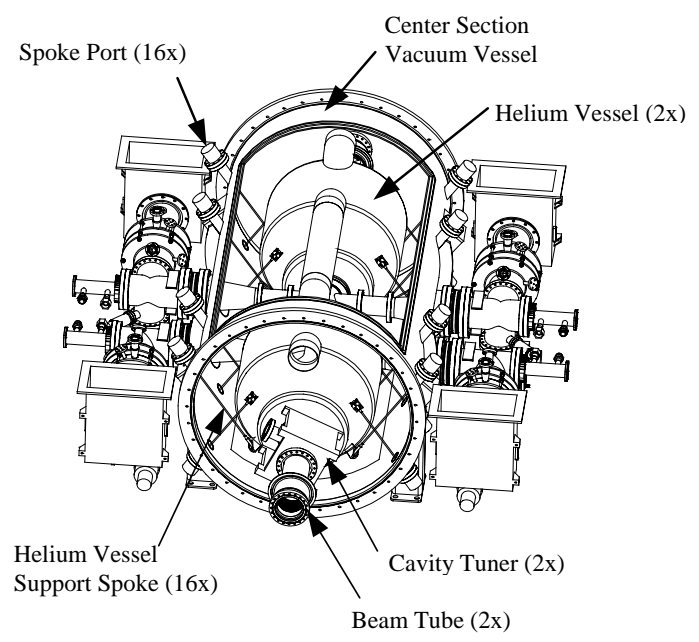

Figure 2: Center section without shields

\section{VACUUM TANK}

\subsection{Vacuum Tank Description}

The $\beta=0.64$ cryomodule vacuum tank is a cylinder which is composed of a center section, spool pieces, and two domed ends, as shown in Figure 1. The center section has two large openings, 120 degrees on the top and 90 degrees on the bottom, to improve access to the interior of the tank for installation of the helium vessels, beam piping, and shields, as shown in Figure 2. The access openings also allow for laminar air flow in the clean room. Once installation of internal parts is complete, access panels are placed over the openings, and a sealing 
skin is placed over the panels. An o-ring surrounding the openings creates a vacuum-tight seal with the sealing skin. The design was adopted from the CERN-LEP cryomodule.

\subsection{Vacuum Tank Analysis}

The vacuum tank components were analyzed for stress and buckling. The center section analysis is more involved, based upon the unusual geometry, as well as the fact that it must perform other functions. These functions include supporting the helium vessels (via spokes mounted on the tank wall) and allowing an interface to the power couplers. The access panels and skins carry the pressure load and create a seal, respectively. The panels carry the pressure load by transmitting hoop stress and longitudinal stress throughout the cylinder, as if it were a continuous cylinder without interruption. The buckling strength of the center section was also analyzed. Hoop stress and longitudinal stress are carried across the panel to center section interface due to the fact that these stresses are compressive. Bending stress cannot be carried across the center section to panel interface. This interface flexibility reduces the buckling strength of the center section under vacuum. The center section/panel/skin geometry was analyzed for buckling in several different ways using both hand calculations and finite element (COSMOS) analyses. The minimum factor of safety obtained was 3 , while much higher factors were obtained for other cases.

\section{HELIUM VESSEL SUPPORT}

\subsection{Helium Vessel Support Arrangement}

The helium vessel is supported from the vacuum tank center section with spokes. There are four spokes at each end of a helium vessel, placed in a radial arrangement to restrain the helium vessel in the radial direction, as shown in Figure 3. The cavities are aligned at room temperature and it is very desirable that this alignment not change after cooldown and thermal equilibrium is reached. In addition to maintaining alignment, the spokes were arranged to minimize loads and stresses in the spokes and helium vessels, and to minimize heat leak to the liquid helium. Two key geometrical features were incorporated into the spoke design that maintain cavity alignment. First, all of the spoke angles to vertical were maintained identical. This ensures that the forces in the vertical direction balance as spoke forces increase during cooldown, as the result of thermal shrinkage strain. The differing spoke lengths have the same thermal shrinkage strain, and will thus have the same stress and force. Accordingly, the beam centerline will not move because of a force imbalance in the vertical direction. Second, a 90 degree angle is maintained between the spoke, and an imaginary radial line which connects the spoke attachment point on the helium vessel to the beam centerline, as shown in Figure 3. Under the premise that the beam centerline remains motionless during cooldown, all points on the helium vessel migrate towards the beam centerline during cooldown. With the 90 degree angle maintained, the helium vessel shrinkage has no effect on spoke force, since the cold end of the spoke translates towards the beam centerline, neither lengthening nor shortening the spoke. There is then no effect on spoke

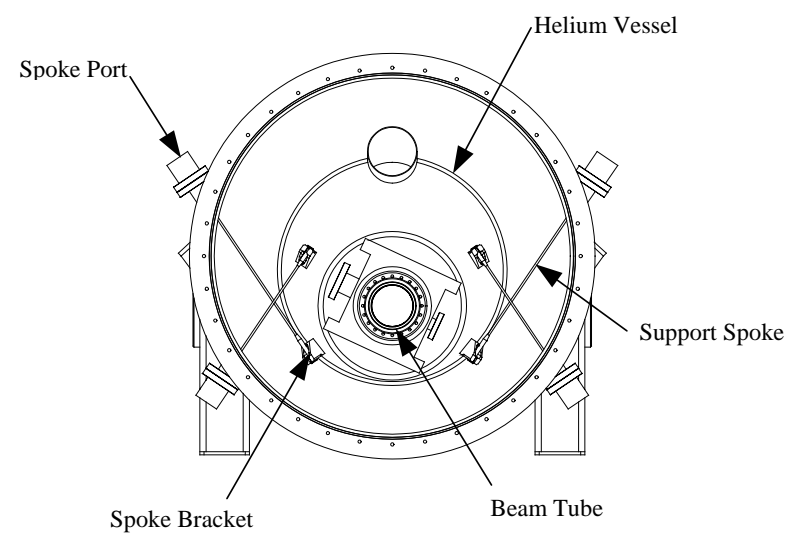

Figure 3: Support spoke arrangement

force from helium vessel shrinkage during cooldown. Analysis of the thermally-induced displacements showed that this arrangement maintains cavity alignment after thermal equilibrium has been reached.

The spokes were placed at an angle to vertical which provides sufficient load carrying capability in both the vertical and horizontal directions. The spokes are 0.25 inches in diameter and will be made of Nitronic stainless steel. Belleville springs will be placed at the warm ends of the spokes to absorb a portion of the thermal shrinkage of the spokes, as shown in Figure 4. Three disc springs will be stacked in series at the warm end of the upper spokes, while two disc springs will be stacked in series at the warm end of the lower spokes. The springs will be

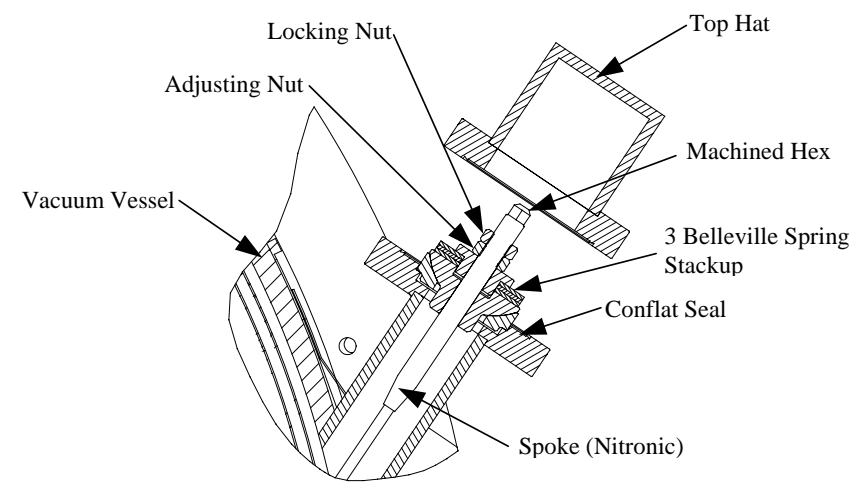

Figure 4: Belleville spring detail 
made of either 17/7 PH or 17/4 PH stainless steel. Upon room temperature alignment of the helium vessel, all spring sets will be partially compressed. At operating temperatures, springs are fully compressed and the spoke system is "solid." The results are summarized in Table 1.

Table 1: Spoke Forces \& Stresses

\begin{tabular}{|l|c|c|c|c|}
\hline & \multicolumn{2}{|c|}{ Room Temp } & \multicolumn{2}{c|}{ Operating Temp } \\
\hline With Springs & $450 \mathrm{lb}$ & $9170 \mathrm{psi}$ & $2250 \mathrm{lb}$ & $46 \mathrm{kpsi}$ \\
\hline Without Springs & $450 \mathrm{lb}$ & $9170 \mathrm{psi}$ & $4060 \mathrm{lb}$ & $83 \mathrm{kpsi}$ \\
\hline
\end{tabular}

\section{POWER COUPLER THERMAL ANALYSIS}

The cryomodule heat loads to the low-temperature helium coolant are dominated by the RF loads in the superconducting niobium cavities. In the $\beta=0.64$ cryomodule, the cavity heat loads account for over $50 \%$ of the total room temperature refrigeration input power. Some of the other contributors to the heat loads are the power couplers, thermal shield, tuner levers, support structure, beamtube, valves, bayonets, and instrumentation. After the cavities, the next biggest contributor to the heat load is the power coupler.

The thermal analysis of the power coupler naturally divides into separate consideration of the inner and outer conductors. Generally, the inner conductor analysis focuses on demonstrating adequate thermal stability against the RF losses from both traveling and standing waves, with some margin added. The outer conductor represents a thermal short connecting room temperature to the $2-\mathrm{K}$ cavity-cooling system. Thermal intercepts are used to remove outer conductor heat loads at temperatures between room temperature and $2 \mathrm{~K}$. Thus, the outer conductor thermal analysis is aimed at minimizing the room-temperature refrigeration input power necessary to offset the heat loads at lower temperatures. The thermal intercepts must be chosen consistent with the refrigerator and cryomodule design.

A 200-node, axisymmetric model using the finite difference approximation was made to describe the inner and outer conductors. The model includes gray body; diffuse, infra-red radiation exchange; conduction through stainless steel, copper, and niobium; RF heating (traveling or standing wave power distributions); and forced convection cooling of the inner and outer conductors. Detailed results for the inner and outer conductor have been published [2]. The inner conductor is found to be adequately cooled for both traveling and standing waves by using $300-\mathrm{K}$ helium at 1.2 atmospheres pressure flowing at $3 \mathrm{~g} / \mathrm{s}$ through a 3-mm annular space, between a stainless-steel sleeve within the copper inner conductor.

The outer conductor cooling approaches considered both distributed counter-flow and localized thermal intercepts. The selected cooling scheme was the result of an extensive trade-study which considered: integration of the power coupler cooling into the cryomodule; impact on the cryo-plant and distribution system; room temperature refrigeration input power; and manufacturability, maintainability, and reliability issues. The selected configuration is a double-point thermal intercept in which the low temperature thermal intercept inlet fluid is $4.6 \mathrm{~K}$, 12 bar helium. After exiting the low temperature intercept, the supercritical helium is used to intercept heat loads from the beam tube, thermal shield, and structural supports, and then circulated through the power coupler high-temperature intercept. The fluid is then returned to the cryo-plant at about $30 \mathrm{~K}$.

\section{STATUS}

A final design review is scheduled for April of this year for the $\beta=0.64$ ED\&D cryomodule. Detailed drawings are being prepared to send to vendors for bids in mid-July, with fabrication of the two cavity $\beta=0.64$ ED\&D cryomodule scheduled for the Fall of 1999.

\section{REFERENCES}

[1] B.M. Campell et al., "Engineering Design of the APT Cryomodules," Proceedings of LINAC98, Chicago, Aug 24-28, 1998

[2] J.A. Waynert et al., "A Thermal Analysis and Optimization of the APT $210 \mathrm{~kW}$ Power Coupler," Proceedings of LINAC98, Chicago, Aug 24-28, 1998 